\title{
TRAVELING WAVES OF A GO-OR-GROW MODEL OF GLIOMA GROWTH*
}

\author{
TRACY L. STEPIEN ${ }^{\dagger}$, ERICA M. RUTTER ${ }^{\ddagger}$, AND YANG KUANG ${ }^{\S}$
}

\begin{abstract}
Glioblastoma multiforme is a deadly brain cancer in which tumor cells excessively proliferate and migrate. The first mathematical models of the spread of gliomas featured reactiondiffusion equations, and later an idea emerged through experimental study called the "Go or Grow" hypothesis in which glioma cells have a dichotomous behavior: a cell either primarily proliferates or primarily migrates. We analytically investigate an extreme form of the "Go or Grow" hypothesis where tumor cell motility and cell proliferation are considered as separate processes. Different solution types are examined via approximate solution of traveling wave equations, and we determine conditions for various wave front forms.
\end{abstract}

Key words. glioblastoma, tumor growth, go or grow, traveling wave, mathematical modeling

AMS subject classifications. 92C50, 35C07, 35K57

DOI. $10.1137 / 17 \mathrm{M} 1146257$

1. Introduction. In this paper, we study the speed and shape of traveling wave solutions of

$$
\begin{aligned}
& \frac{\partial M}{\partial t}=\underbrace{D \nabla^{2} M}_{\text {diffusion }}+\underbrace{\Phi_{M}(M, P)}_{\text {net growth/death }}+\underbrace{\lambda_{P \rightarrow M}(T) P-\lambda_{M \rightarrow P}(T) M}_{\text {transitions between } M \text { and } P}, \\
& \frac{\partial P}{\partial t}=\underbrace{\Phi_{P}(M, P)}_{\text {net growth/death }}-\underbrace{\lambda_{P \rightarrow M}(T) P+\lambda_{M \rightarrow P}(T) M}_{\text {transitions between } M \text { and } P},
\end{aligned}
$$

where $M(\mathbf{x}, t)$ and $P(\mathbf{x}, t)$ are variables representing the density of two subpopulations such that $T=M+P$ is the total population density and members of one subpopulation may transition into becoming a member of the other subpopulation.

This system of equations describes the spreading of glioblastoma multiforme (GBM), a deadly brain cancer which is characterized by extremely diffusive and proliferative behavior (Norden and Wen [21]). Mathematical modeling of this cancer began in the 1990s with a focus on describing the spreading of cancer cells via reaction-diffusion equations (see the review paper by Martirosyan et al. [19] and the references therein); however, later an idea emerged through experimental study that glioblastoma cells had a dichotomous behavior: either an individual cell proliferates rapidly and migrates slowly, or the cell migrates rapidly and proliferates slowly

\footnotetext{
*Received by the editors September 5, 2017; accepted for publication (in revised form) March 12, 2018; published electronically June 20, 2018.

http://www.siam.org/journals/siap/78-3/M114625.html

Funding: The third author's work was partially supported by NSF grants DMS-1518529 and DMS-1615879.

†School of Mathematical and Statistical Sciences, Arizona State University, Tempe, AZ 85287. Current address: Department of Mathematics, University of Arizona, Tucson, AZ 85719 (stepien@math.arizona.edu).

${ }^{\ddagger}$ School of Mathematical and Statistical Sciences, Arizona State University, Tempe, AZ 85287. Current address: Center for Research in Scientific Computation, Department of Mathematics, North Carolina State University, Raleigh, NC 27695 (erutter@ncsu.edu).

$\S$ School of Mathematical and Statistical Sciences, Arizona State University, Tempe, AZ 85287 (kuang@asu.edu).
} 
(Giese et al. [8, 9, 10], Godlewski et al. [11]). Generally, cells that proliferate rapidly are located in the central core of the tumor, and cells that migrate rapidly are located at the peripheral edges of the tumor. The transformation of a cell from one population to the other is triggered by a phenotypic switch, which could be due to a variety of mechanisms. This idea was termed the "Go or Grow" hypothesis (Hatzikirou et al. [12]).

In our previous work, we have investigated the applicability of a single-equation reaction-diffusion equation model (Rutter et al. [24]) and a single-equation densitydependent diffusion model (Stepien et al. [31]) to experimental data as well as analyzed the existence of traveling wave solutions in the latter paper. Stepien et al. [31] and others such as Scribner and Fathallah-Shaykh [26] have shown that incorporating more complex dynamics into a single equation model can result in behavior characteristic of GBM invasion. Here, we analytically investigate an extreme form of the "Go or Grow" hypothesis in which there is no proliferation term for the migrating cells and no diffusion for the proliferating cells, which is based in part on the tumor cord growth model of Thalhauser et al. [32] and in part on biological findings that glioma cells generally migrate in fast bursts followed by rest periods in which they proliferate (Farin et al. [6]), as well as inspired by the many glioma growth differential equation models that incorporate the "Go or Grow" hypothesis (e.g. Chauviere et al. [4], Gerlee and Nelander [7], Martínez-González et al. [18], Pham et al. [22], Saut et al. [25], Stein et al. [30]).

To study the specific case of glioma growth from the general form of the model (1), let $M$ be the density of migrating cells and $P$ be the density of proliferating cells. We specify the net growth/death functions as

$$
\begin{aligned}
& \Phi_{M}(M, P)=-\mu M, \\
& \Phi_{P}(M, P)=g\left(1-\frac{T}{T_{\max }}\right) P
\end{aligned}
$$

and transition functions as

$$
\begin{aligned}
& \lambda_{P \rightarrow M}(T)=\epsilon k \frac{T^{n}}{T^{n}+K_{M}^{n}}, \\
& \lambda_{M \rightarrow P}(T)=k \frac{K_{P}^{n}}{T^{n}+K_{P}^{n}},
\end{aligned}
$$

resulting in the final form of the model that we analyze in this paper,

$$
\begin{aligned}
& \frac{\partial M}{\partial t}=D \nabla^{2} M+\epsilon k \frac{T^{n}}{T^{n}+K_{M}^{n}} P-k \frac{K_{P}^{n}}{T^{n}+K_{P}^{n}} M-\mu M, \\
& \frac{\partial P}{\partial t}=g\left(1-\frac{T}{T_{\max }}\right) P-\epsilon k \frac{T^{n}}{T^{n}+K_{M}^{n}} P+k \frac{K_{P}^{n}}{T^{n}+K_{P}^{n}} M .
\end{aligned}
$$

Taking the spatial domain to be infinite, appropriate boundary conditions are $\nabla M(R, t)=\nabla P(R, t)=0$ as radius $R \rightarrow \infty$, and an appropriate initial condition is one that has a finite density of cells for both $M$ and $P$ and compact support. It is assumed that all the parameters $\left(D, \epsilon, k, K_{M}, K_{P}, \mu, g, T_{\max }\right)$ are positive.

In section 2 we analyze the speed of traveling waves of the system (4), and in section 3 we analyze the shape of traveling waves of the system before summarizing our results in section 4 . 
2. Traveling wave and its speed. A traveling wave solution of (4) is a solution of the form

$$
M(\mathbf{x}, t)=U(\xi), \quad P(\mathbf{x}, t)=V(\xi), \quad \xi=\mathbf{r} \cdot \mathbf{x}-c t,
$$

where $c \geq 0$ is the speed of the traveling wave, $\mathbf{r}$ is the propagating direction, and functions $U$ and $V$ are defined on the interval $(-\infty,+\infty)$. For convenience, define

$$
T(\mathbf{x}, t)=W(\xi)
$$

since $T=M+P$ is the total cell density, and so $W=U+V$. Substitution of forms (5)-(6) into (4) gives the following system of ordinary differential equations:

$$
\begin{gathered}
c U^{\prime}+r D U^{\prime \prime}+\epsilon k \frac{(U+V)^{n}}{(U+V)^{n}+K_{M}^{n}} V-k \frac{K_{P}^{n}}{(U+V)^{n}+K_{P}^{n}} U-\mu U=0, \\
c V^{\prime}+g\left(1-\frac{U+V}{T_{\max }}\right) V-\epsilon k \frac{(U+V)^{n}}{(U+V)^{n}+K_{M}^{n}} V+k \frac{K_{P}^{n}}{(U+V)^{n}+K_{P}^{n}} U=0,
\end{gathered}
$$

where $r=|\mathbf{r}|^{2}$ and the prime ' denotes differentiation with respect to $\xi$.

We begin with considering the speed $c$ of the traveling wave solutions satisfying (7). Substituting the ansatz

$$
U(\xi)=\hat{U} e^{-\theta \xi}, \quad V(\xi)=\hat{V} e^{-\theta \xi}
$$

into (7) we obtain

$$
\begin{aligned}
&-c \theta \hat{U}+r D \theta^{2} \hat{U}+\epsilon k \frac{(\hat{U}+\hat{V})^{n} e^{-n \theta \xi}}{(\hat{U}+\hat{V})^{n} e^{-n \theta \xi}+K_{M}^{n}} \hat{V} \\
&-k \frac{K_{P}^{n}}{(\hat{U}+\hat{V})^{n} e^{-n \theta \xi}+K_{P}^{n}} \hat{U}-\mu \hat{U}=0, \\
&-c \theta \hat{V}+g\left(1-\frac{(\hat{U}+\hat{V}) e^{-\theta \xi}}{T_{\max }}\right) \hat{V}-\epsilon k \frac{(\hat{U}+\hat{V})^{n} e^{-n \theta \xi}}{(\hat{U}+\hat{V})^{n} e^{-n \theta \xi}+K_{M}^{n}} \hat{V} \\
&+k \frac{K_{P}^{n}}{(\hat{U}+\hat{V})^{n} e^{-n \theta \xi}+K_{P}^{n}} \hat{U}=0 .
\end{aligned}
$$

Then linearizing ahead of the wave front (about $U=V=0$, when $\xi \rightarrow \infty$ ) to leading order gives

$$
\begin{gathered}
\left(-c \theta+r D \theta^{2}-k-\mu\right) \hat{U}=0, \\
c \theta \hat{V}=g \hat{V}+k \hat{U} .
\end{gathered}
$$

Solving (10a) for $c$, we obtain the dispersion relation

$$
c=r D \theta-\frac{k+\mu}{\theta} .
$$

The derivative of (11) with respect to $\theta$ is positive, which implies that $c$ as a function of $\theta$ is always increasing and therefore does not have any local minima. Thus the dispersion relation does not give a condition for the minimum wave speed.

Copyright $@$ by SIAM. Unauthorized reproduction of this article is prohibited. 
Solving (10b) for $c$, we obtain

$$
c=\frac{1}{\theta}\left(g+k \frac{\hat{U}}{\hat{V}}\right) .
$$

Since the density of migrating calls $M$ and proliferating cells $P$ is nonnegative, then by (8), both $\hat{U}$ and $\hat{V}$ must be nonnegative. Substituting expression (12) for $c$ into (10a) and using the fact that $\frac{\hat{U}}{\hat{V}} \geq 0$,

$$
r D \theta^{2}=g+k\left(\frac{\hat{U}}{\hat{V}}+1\right)+\mu \geq g+k+\mu,
$$

which implies that the minimum value that $\theta$ can attain is

$$
\theta_{\min }=\sqrt{\frac{g+k+\mu}{r D}}
$$

Hence, substituting $\theta_{\min }$ into the dispersion relation (11) gives the minimum wave speed

$$
c \geq c_{\min }=g \sqrt{\frac{r D}{g+k+\mu}} .
$$

Thus, the range of wave speeds for equation (4) is satisfied by (15).

To compare the analytical wave speed (15) with the numerically observed wave speed, we ran simulations using the MATLAB function PDEPE over a substantially large spatial domain $(x \in[-20,20])$ and time domain $(t \in[0,50])$. The MATLAB function POLYFIT was used to fit the slope of the spatial position of the tracking density $0.1 \times T_{\max }$ as time increased, starting at $t=25$ to ensure the traveling wave solutions were established. The calculation was performed separately for the migrating cells, the proliferating cells, and the total number of cells, and it was found that the wave speeds were equal for almost all cell populations, supporting the assumption in (5). For small values of $k$ and $g$, we noticed a underestimation of the wave speed for the migrating cells. This may be due to the choice of our tracking density in POLYFIT, as these parameter choices may result in very low cell density levels for the migrating population.

The results of these calculations are presented in Figure 1. The minimum wave speed $c_{\min }(15)$ is represented by the red dashed line. The numerically observed wave speed for the migrating population is given by blue plusses, the numerically observed wave speed for the proliferating population is given by green triangles, and the numerically observed wave speed for the total population is in black asterisks. Our results indicate that the numerically observed wave speed is generally greater than or equal to the analytical minimum wave speed. There are a few data points for very small $k$ values that fall below the analytical wave speed range, in particular for the migrating population. This may also be related to the choice of the tracking density in POLYFIT. Unlike other numerical wave speed calculations, our calculations lie slightly above the analytical wave speed. This may be due to the linearization process eliminating higher-order terms which may influence the wave speed. 

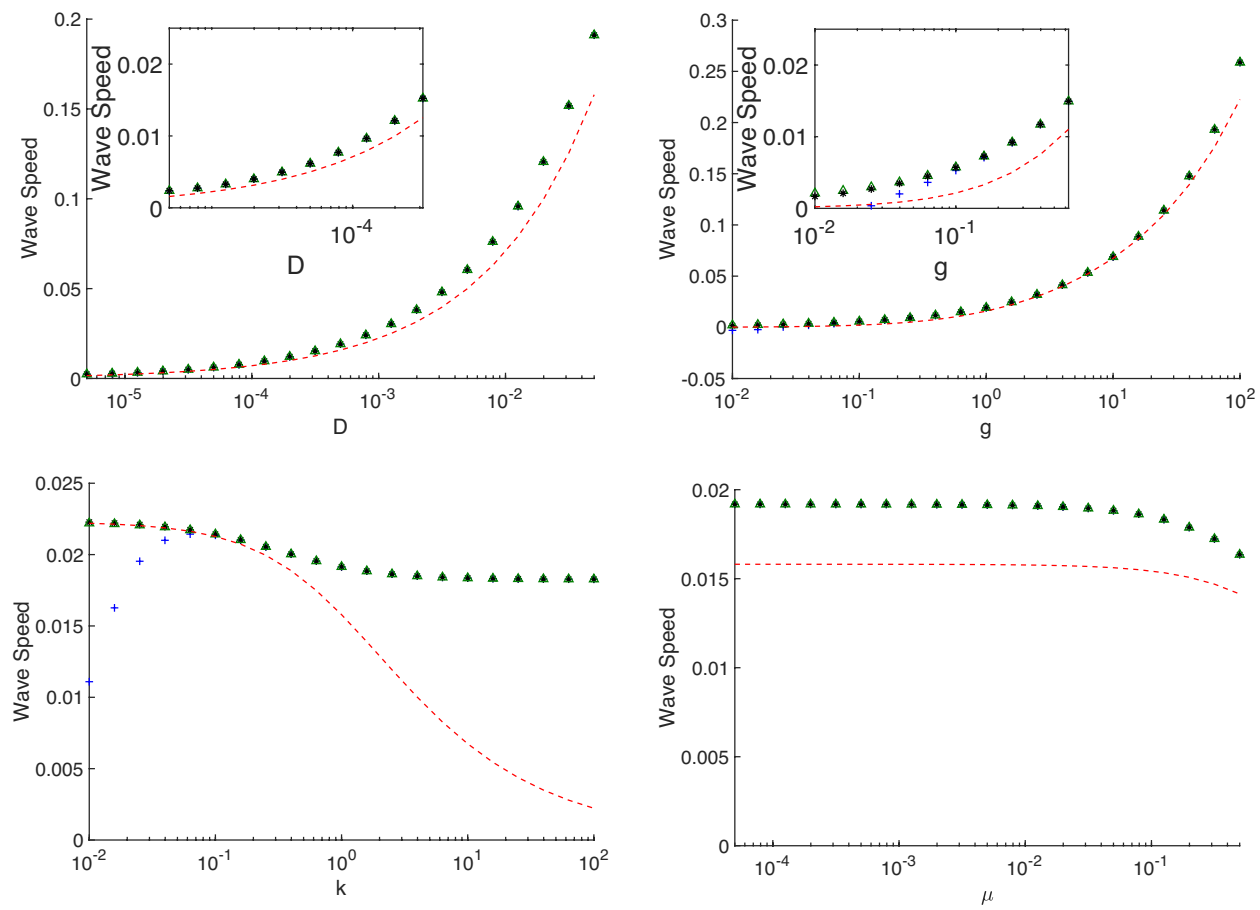

FIG. 1. Comparison of the analytical and numerical wave speeds. The red dashed line represents the analytical wave speed (15). The blue plusses represent the numerically observed migrating wave speed, green triangles represent the numerically observed proliferating wave speed, and black asterisks represent the numerically observed total wave speed. The base parameters are $D=5 \times 10^{-4}, g=1$, $k=1, \mu=0.005, K_{m}=K_{p}=0.5$, and $\epsilon=1$.

3. Approximate traveling wave. Besides the wave speed of traveling wave solutions of (4), we are also interested in the shape of the traveling waves. Here we use a method developed by Canosa [2] that has been used to analyze other models of cancer growth (Sherratt [27], Sherratt and Chaplain [28], Zhu et al. [36], Quinn and Sinkala [23], Zhu and $\mathrm{Ou}$ [35]) and other types of cell migration such as in wound healing and embryonic development (Dale et al. [5], Newgreen et al. [20], Simpson et al. [29], Landman et al. [14], Cai et al. [1], Trewenack and Landman [33]). This method gives a good approximation to the solution of the dimensionless FisherKolmogorov equation $u_{t}=u_{x x}+u(1-u)$, and here we examine the agreement between the approximation of (4) that we obtain using the method of Canosa [2] to numerical simulations.

To obtain an approximation of the traveling wave solution via the method of Canosa [2], we rescale the traveling wave coordinate by writing $z=-\frac{1}{c} \xi$. With this change of variables, system (7) becomes

$$
\begin{gathered}
-\frac{d U}{d z}+\frac{r D}{c^{2}} \frac{d^{2} U}{d z^{2}}+\epsilon k \frac{(U+V)^{n}}{(U+V)^{n}+K_{M}^{n}} V-k \frac{K_{P}^{n}}{(U+V)^{n}+K_{P}^{n}} U-\mu U=0 \\
-\frac{d V}{d z}+g\left(1-\frac{U+V}{T_{\max }}\right) V-\epsilon k \frac{(U+V)^{n}}{(U+V)^{n}+K_{M}^{n}} V+k \frac{K_{P}^{n}}{(U+V)^{n}+K_{P}^{n}} U=0 .
\end{gathered}
$$

Take $\delta=\frac{1}{c^{2}}$ to be a perturbation parameter, and consider a sufficiently large wave speed such that $c \geq c_{\min }$ (15). Biologically, we expect the transition rate $k$ and the 
death rate $\mu$ to be small, so $c_{\min }=\mathcal{O}(\sqrt{D g})$. Since GBM is highly proliferative and infiltrative such that cancer cells can disperse widely throughout the brain, $c_{\min }$ may be large during certain stages of cancer. For example, taking units of speed as mm/y, GBM patient velocity data as reported in Wang et al. [34] supports the assumption that $\delta$ is small.

Substituting into the previous equation the regular perturbation expansions

$$
U(\xi ; \delta)=\sum_{r=0}^{\infty} U_{r} \delta^{r}, \quad V(\xi ; \delta)=\sum_{r=0}^{\infty} V_{r} \delta^{r},
$$

we investigate the lowest order terms $U_{0}$ and $V_{0}$ which satisfy

$$
\begin{aligned}
& \frac{d U}{d z}=\epsilon k \frac{(U+V)^{n}}{(U+V)^{n}+K_{M}^{n}} V-k \frac{K_{P}^{n}}{(U+V)^{n}+K_{P}^{n}} U-\mu U, \\
& \frac{d V}{d z}=g\left(1-\frac{U+V}{T_{\max }}\right) V-\epsilon k \frac{(U+V)^{n}}{(U+V)^{n}+K_{M}^{n}} V+k \frac{K_{P}^{n}}{(U+V)^{n}+K_{P}^{n}} U,
\end{aligned}
$$

where the subscripts on $U_{0}$ and $V_{0}$ have been omitted for notational simplicity.

It is more convenient to rewrite the system as one in $W(6)$ and $V$, which is

$$
\begin{aligned}
\frac{d W}{d z} & =g\left(1-\frac{W}{T_{\max }}\right) V-\mu(W-V), \\
\frac{d V}{d z} & =g\left(1-\frac{W}{T_{\max }}\right) V-\epsilon k \frac{W^{n}}{W^{n}+K_{M}^{n}} V+k \frac{K_{P}^{n}}{W^{n}+K_{P}^{n}}(W-V) .
\end{aligned}
$$

We look for a solution with $(W, V)=(0,0)$ (which is an equilibrium point of (19)) as $z \rightarrow-\infty$, since this corresponds to $x \rightarrow \infty$, ahead of the wave. In subsection 3.1 we examine the stability of this equilibrium point and the nullclines of the system, in subsection 3.2 we construct a positively invariant region $\Omega$, in subsection 3.3 we show that no periodic orbits exist in $\Omega$, in subsection 3.4 we show that there is nonempty intersection between the solution that tends to $(0,0)$ as $z \rightarrow-\infty$ and $\Omega$, and in subsection 3.5 we examine the interior fixed points in $\Omega$. These steps will lead to the conclusion of the existence of a heteroclinic orbit corresponding to the approximate traveling wave under certain conditions.

3.1. Equilibrium point at origin and nullclines. The components of the Jacobian are

$$
\begin{aligned}
J_{11}(W, V) & =-\frac{g}{T_{\max }} V-\mu, \\
J_{12}(W, V) & =g\left(1-\frac{W}{T_{\max }}\right)+\mu, \\
J_{21}(W, V) & =-\frac{g}{T_{\max }} V-\epsilon k V\left[\frac{\left(W^{n}+K_{M}^{n}\right) n W^{n-1}-n W^{n}}{\left(W^{n}+K_{M}^{n}\right)^{2}}\right] \\
\quad+k\left[\frac{K_{P}^{n}}{W^{n}+K_{P}^{n}}+(W-V) \frac{K_{P}^{n}}{\left(W^{n}+K_{P}^{n}\right)^{2}} n W^{n-1}\right], & \\
J_{22}(W, V) & =g\left(1-\frac{W}{T_{\max }}\right)-\epsilon k \frac{W^{n}}{W^{n}+K_{M}^{n}}-k \frac{K_{P}^{n}}{W^{n}+K_{P}^{n}},
\end{aligned}
$$

and thus at the equilibrium point $(W, V)=(0,0)$, the Jacobian is

$$
J(0,0)=\left(\begin{array}{cc}
-\mu & g+\mu \\
k & g-k
\end{array}\right) .
$$

Copyright $@$ by SIAM. Unauthorized reproduction of this article is prohibited. 
The eigenvalues of $J(0,0)$ are

$$
\lambda_{+}=g>0, \quad \lambda_{-}=-(k+\mu)<0,
$$

implying that the equilibrium point $(0,0)$ is a saddle. The corresponding eigenvectors are

$$
\mathbf{v}_{+}=\left(\begin{array}{l}
1 \\
1
\end{array}\right), \quad \mathbf{v}_{-}=\left(\begin{array}{c}
\frac{-(g+\mu)}{k} \\
1
\end{array}\right),
$$

and thus a traveling wave solution will correspond to the trajectory leaving $(0,0)$ along the $\mathbf{v}_{+}$eigenvector.

The $W$-nullcline is

$$
V(W)=\frac{\mu W}{g\left(1-\frac{W}{T_{\max }}\right)+\mu},
$$

and since its derivative is

$$
V^{\prime}(W)=\frac{\mu(g+\mu)}{\left(g\left(1-\frac{W}{T_{\max }}\right)+\mu\right)^{2}}>0,
$$

then the $W$-nullcline is a strictly increasing function. Furthermore, $V(0)=0$, and there is a vertical asymptote at $W=T_{\max }\left(1+\frac{\mu}{g}\right)>T_{\max }$.

The $V$-nullcline is

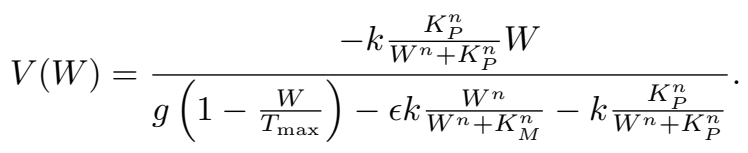

We conjecture that the $V$-nullcline is a strictly decreasing function for $g \geq \epsilon k$; see Figure 2 .

3.2. Positively invariant region. If we extend the linearized eigenvector $\mathbf{v}_{+}$ (23) as a line and consider the region $\Omega$ bound by that line, the horizontal $W$-axis, and the line $W=T_{\max }$, as illustrated in Figure 3, we claim that the unstable manifold of the saddle at $(0,0)$ is trapped within the region $\Omega$.

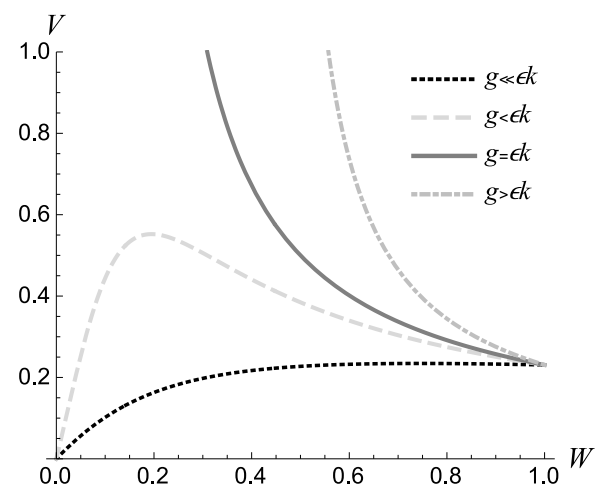

FIG. 2. Plot of the $V$-nullcline for various values of $g$. Other parameter values are $n=1$, $T_{\max }=1, \epsilon=1, k=0.5, \mu=0.25, K_{P}=0.25, K_{M}=0.5$. 


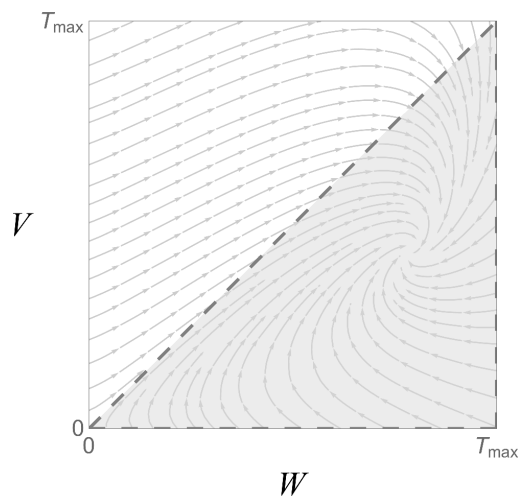

FIG. 3. Positively invariant region $\Omega$ shaded in gray as described in subsection 3.2. The dashed lines correspond to the linearized eigenvector $\mathbf{v}_{+}(23)$, line $V=0$, and line $W=T_{\max }$. The vector field of a typical system is shown with arrows.

Lemma 1. Let $\Omega$ be the open region bounded by the lines $\{(W, V): V=0\}$, $\left\{(W, V): W=T_{\max }\right\}$, and $\{(W, V): V=W\} . \Omega$ is positively invariant.

Proof. Along the line $\{(W, V): V=0\}$, we have

$$
\begin{aligned}
& \frac{d W}{d t}=-\mu W<0, \\
& \frac{d V}{d t}=\frac{k K_{P}^{n} W}{W^{n}+K_{P}^{n}}>0,
\end{aligned}
$$

so the flow is up and to the left across the line.

Along the line $\left\{(W, V): W=T_{\max }\right\}$, we have

$$
\begin{aligned}
& \frac{d W}{d t}=-\mu\left(T_{\max }-V\right)<0 \quad \text { if } V<T_{\max }, \\
& \frac{d V}{d t}=k \frac{K_{P}^{n}}{T_{\max }^{n}+K_{P}^{n}}\left(T_{\max }-V\right)-\epsilon k \frac{T_{\max }^{n}}{T_{\max }^{n}+K_{M}^{n}} V,
\end{aligned}
$$

so the flow is to the left across the line.

Along the line $\{(W, V): V=W\}$ which has slope 1 , the slope of the vector field is

$$
1-\frac{\epsilon k \frac{V^{n}}{V^{n}+K_{M}^{n}}}{g W\left(1-\frac{W}{T_{\max }}\right)}<1 \quad \text { if } W<T_{\max },
$$

and since

$$
\begin{aligned}
& \frac{d W}{d t}=g W\left(1-\frac{W}{T_{\max }}\right)>0 \quad \text { if } W<T_{\max }, \\
& \frac{d V}{d t}=g W\left(1-\frac{W}{T_{\max }}\right)-\epsilon k \frac{W^{n+1}}{W^{n}+K_{M}^{n}}
\end{aligned}
$$

the flow is to the right and at a slope less than 1 , so it crosses the line to the inside of the triangular region.

The corner $(W, V)=(0,0)$ is an equilibrium point, so the flow cannot leave through it. At the corner $(W, V)=\left(T_{\max }, T_{\max }\right)$, the flow is directly downward, and 
at the corner $(W, V)=\left(T_{\max }, 0\right)$, the flow is up and to the left. Thus, since the flow points inward on the boundary of $\Omega$ and all trajectories are confined, $\Omega$ is positively invariant.

3.3. No periodic orbits. To rule out the existence of periodic orbits within the positively invariant region $\Omega$, we invoke Dulac's criterion. Let $f_{1}(W, V)$ be the right-hand side of $(19 \mathrm{a})$ and $f_{2}(W, V)$ be the right-hand side of $(19 \mathrm{~b})$.

Theorem 2 (Dulac's criterion). Let $B(W, V)$ be $\mathbf{C}^{1}$ on a simply connected region $\Omega \subset \mathbb{R}^{2}$. If $\frac{\partial\left(B f_{1}\right)}{\partial W}+\frac{\partial\left(B f_{2}\right)}{\partial V}$ is not identically zero and does not change sign in $\Omega$, then (19) has no closed orbits lying entirely in $\Omega$.

If we set

$$
B(W, V)=\frac{1}{V}
$$

then

$$
\frac{\partial\left(B f_{1}\right)}{\partial W}+\frac{\partial\left(B f_{2}\right)}{\partial V}=-\frac{k K_{P}^{n} T_{\max } W+\left(W^{n}+K_{P}^{n}\right)\left(g V+\mu T_{\max }\right) V}{T_{\max }\left(W^{n}+K_{P}^{n}\right) V^{2}}<0,
$$

so the expression is of one sign within the positively invariant region $\Omega$, and therefore there are no periodic orbits within the closed positively invariant region $\bar{\Omega}$.

3.4. Nonempty intersection. The linearized eigenvector of the unstable manifold $\mathbf{v}_{+}(23)$ coincides with the line $\{(W, V): V=W\}$, which is part of the boundary of the positively invariant region $\Omega$. Since trajectories that leave the point $(0,0)$ in the region $\Omega$ have the tangent vector $\mathbf{v}_{+}$at $(0,0)$, and since the flow is to the right across the line $\{(W, V): V=W\}$, trajectories leaving $(0,0)$ must leave tangentially to the right of $\mathbf{v}_{+}$. Therefore, the unstable manifold of the saddle at $(0,0)$ has nonempty intersection with $\Omega$.

The unstable manifold of the saddle $(0,0)$ thus remains in $\Omega$ for all time, and hence the $\omega$-limit set of the corresponding orbit is also contained in $\Omega$. Thus far, we have not shown how many equilibrium points are in the interior of $\Omega$, but there will be a heteroclinic orbit that connects the equilibrium point $(0,0)$ with some interior equilibrium point, corresponding with the approximate traveling wave solution. The behavior of the dynamical system and which equilibrium point is in the $\omega$-limit set will determine the shape of the approximate traveling wave profile.

3.5. Interior equilibrium points. One may verify that the only equilibrium point on the boundary of the positively invariant triangular region $\partial \Omega$ is $(W, V)=$ $(0,0)$. Recall that this equilibrium point is a saddle and the linearized eigenvector corresponding to the unstable manifold $\mathbf{v}_{+}$intersects $\bar{\Omega}$ while the linearized eigenvector corresponding to the stable manifold $\mathbf{v}_{-}$does not intersect $\bar{\Omega}$ (subsections 3.1 and 3.2 ). Thus, since there are no periodic orbits (subsection 3.3), there must be at least one interior equilibrium point, as any trajectory that enters $\Omega$ cannot leave $\Omega$.

In the subsections below we examine the cases when $n$ varies to determine the number of interior equilibrium points as well as which equilibrium point is in the $\omega$ limit set of the heteroclinic orbit corresponding with the approximate traveling wave solution. 
3.5.1. Case: $\boldsymbol{n}=\mathbf{0}$. If we set $n=0$, then system (19) becomes

$$
\begin{aligned}
& \frac{d W}{d z}=g\left(1-\frac{W}{T_{\max }}\right) V-\mu(W-V), \\
& \frac{d V}{d z}=g\left(1-\frac{W}{T_{\max }}\right) V-\frac{1}{2} \epsilon k V+\frac{1}{2} k(W-V) .
\end{aligned}
$$

It is straightforward to calculate the equilibrium points of this system, which are $(W, V)=(0,0)$ and

$$
(W, V)=\left(T_{\max } \frac{g(k+2 \mu)-\epsilon k \mu}{g(k+2 \mu)}, \quad T_{\max } \frac{g(k+2 \mu)-\epsilon k \mu}{g(k+\epsilon k+2 \mu)}\right) .
$$

At the origin, the Jacobian matrix when $n=0$ is

$$
J(0,0)=\left(\begin{array}{cc}
-\mu & g+\mu \\
\frac{1}{2} k & g-\frac{1}{2} \epsilon k-\frac{1}{2} k
\end{array}\right),
$$

and thus to still have a saddle at the origin the determinant of $J(0,0)$ must be negative, or equivalently,

$$
\epsilon k \mu<g(k+2 \mu) .
$$

For a nonzero equilibrium point $\left(W^{*}, V^{*}\right)$ to be in $\Omega$, then the following condition must hold:

$$
0<V^{*}<W^{*}<T_{\max } .
$$

Condition (37) for equilibrium point (34) is in fact equivalent to condition (36), under the assumption that all parameters are positive.

If condition (36) is satisfied, then there is one interior equilibrium point (34). The determinant of the Jacobian at this equilibrium point, $\operatorname{det} J=\frac{1}{2}(g(k+2 \mu)-\epsilon k \mu)$, is positive under condition (36), and the trace,

$$
\operatorname{tr} J=-\left(\frac{(g+\mu)(k+2 \mu)}{k+k \epsilon+2 \mu}+\frac{k(k+k \epsilon+2 \mu)}{2(k+2 \mu)}\right),
$$

is negative, so the equilibrium point is an attractor.

The shape of the traveling wave profile is dependent on what kind of attractor the equilibrium point (34) is. In particular, if $(\operatorname{tr} J)^{2}-4 \operatorname{det} J<0$, then the attractor is a stable spiral (Figure 4(a)), and the resulting traveling wave profiles have a prominent bump at the wave front. Oscillations behind the wave fronts decay quickly numerically for all the stable spiral cases we studied, and thus in the region near the wave front, the first bump is the feature that stands out the most. If instead $(\operatorname{tr} J)^{2}-4 \operatorname{det} J>0$, then the attractor is a stable node (Figure 4(b)), and the resulting traveling wave profiles are monotonic and do not have a bump.

The density of the proliferating and migrating cells in the center of the tumor core (as $z \rightarrow \infty$ ) are given by the equilibrium point (34); in particular,

$$
\begin{gathered}
V \stackrel{z \rightarrow \infty}{\longrightarrow} T_{\max } \frac{g(k+2 \mu)-\epsilon k \mu}{g(k+\epsilon k+2 \mu)} \\
U=W-V \stackrel{z \rightarrow \infty}{\longrightarrow} T_{\max }\left(\frac{g(k+2 \mu)-\epsilon k \mu}{g(k+2 \mu)}-\frac{g(k+2 \mu)-\epsilon k \mu}{g(k+\epsilon k+2 \mu)}\right) .
\end{gathered}
$$

Copyright $@$ by SIAM. Unauthorized reproduction of this article is prohibited. 

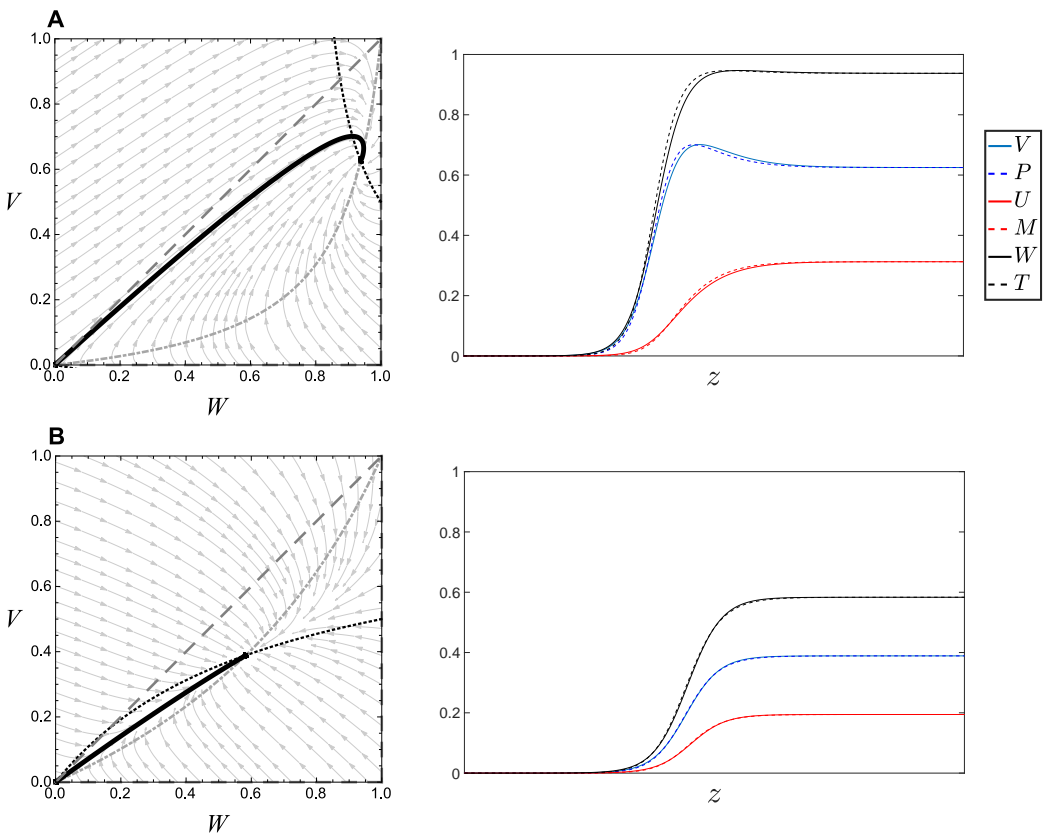

FIG. 4. (Left) Phase portrait of the system (19) with $n=0$ such that condition (36) is satisfied. The dark gray dashed lines represent the boundaries of the positively invariant region $\Omega$ (cf. Figure 3 ), the black dotted curve is the $V$-nullcline, the light gray dash-dotted curve is the $W$-nullcline, and the black solid curve is the unstable manifold of the saddle $(0,0)$. (Right) The corresponding traveling wave profile of the solution trajectory in traveling wave coordinate $z$ (analytical profiles: $V$, $U, W$; numerical profiles: $P, M, T)$. Parameters $T_{\max }=1, \epsilon=1, k=0.5, \mu=0.25, K_{P}=0.25$, $K_{M}=0.5$, and (a) $g=2$, (b) $g=0.3$.

For all the cases we describe within subsection 3.5, we illustratively compare the traveling wave profile that we obtain from the phase portrait analysis ("analytical") to the traveling wave profile that we obtain with a numerical simulation with the same parameter values and an arbitrary, but numerically tractable, value of $D$ ("numerical") in the right-side panels in the figures. The numerical simulation is scaled by a factor of $\frac{1}{c}$, where $c$ is the numerically calculated wave speed, so that both the analytical and numerical traveling wave profiles have $z$ as the independent variable.

3.5.2. Case: $\boldsymbol{n} \rightarrow \infty$. If we take $n \rightarrow \infty$, then the transition functions (3) become approximated by Heaviside functions $H$, and system (19) becomes

$$
\begin{aligned}
& \frac{d W}{d z}=g\left(1-\frac{W}{T_{\max }}\right) V-\mu(W-V), \\
& \frac{d V}{d z}=g\left(1-\frac{W}{T_{\max }}\right) V-\epsilon k H\left(W-K_{M}\right) V+k H\left(K_{P}-W\right)(W-V) .
\end{aligned}
$$

Depending on the relation between $K_{P}$ and $K_{M}$, the interval $\left[0, T_{\max }\right]$ will be split into two or three subintervals where a different system of ordinary differential equations governs the behavior within each subinterval. A solution trajectory of (40) is a piecewise trajectory formed by piecing the solution trajectories in each subinterval together. The three subcases where $K_{P}<K_{M}, K_{P}>K_{M}$, and $K_{P}=K_{M}$ are discussed below. 
3.5.2.1. Subcase: $\boldsymbol{K}_{\boldsymbol{P}}<\boldsymbol{K}_{\boldsymbol{M}}$. If $K_{P}<K_{M}$, (40b) can be rewritten as

$$
\frac{d V}{d z}=g\left(1-\frac{W}{T_{\max }}\right) V+ \begin{cases}k(W-V), & W \in\left[0, K_{P}\right), \\ 0, & W \in\left[K_{P}, K_{M}\right), \\ -\epsilon k V, & W \in\left[K_{M}, T_{\max }\right] .\end{cases}
$$

First subinterval $\left[0, K_{P}\right)$. For the equations defined in the first subinterval, the equilibrium points are $(0,0)$ and $\left(T_{\max }, T_{\max }\right)$. The Jacobian $J(0,0)$ is as given in (21), so the origin is a saddle, and the eigenvalues of the Jacobian $J\left(T_{\max }, T_{\max }\right)$ are $-g$ and $-(k+\mu)$, so $\left(T_{\max }, T_{\max }\right)$ is an attractor.

The slope of the vector field along the line $\{(W, V): V=W\}$ is 1 , which is the same as the slope of the line. Since the unstable manifold of the saddle at $(0,0)$ corresponds with the linearized eigenvector $\mathbf{v}_{+}(23)$, which has a slope of 1 , then the solution trajectory leaving $(0,0)$ along the unstable manifold travels along the line $\{(W, V): V=W\}$ in the first interval from $(0,0)$ to $\left(K_{P}, K_{P}\right)$.

Second subinterval $\left[K_{P}, K_{M}\right)$. For the equations defined in the second subinterval, the equilibrium points are $(0,0)$ and $\left(T_{\max }, T_{\max }\right)$, and the slope of the vector field along the line $\{(W, V): V=W\}$ is 1 , the same as the slope of the line, which is the same result as in the first subinterval. Though the Jacobian $J(0,0)$ is different for the equations defined in the second interval compared to the first subinterval, the eigenpair $\left(\lambda_{+}, \mathbf{v}_{+}\right)(22)-(23)$ is the same, and the origin is still a saddle. Furthermore, $\left(T_{\max }, T_{\max }\right)$ is still an attractor. Thus the solution trajectory in the second interval starts from $\left(K_{P}, K_{P}\right)$ and ends at $\left(K_{M}, K_{M}\right)$ along the line $\{(W, V): V=W\}$.

Third subinterval $\left[K_{M}, T_{\max }\right]$. For the equations defined in the third subinterval, the equilibrium points are $(W, V)=(0,0)$ and

$$
(W, V)=\left(T_{\max }\left(1-\frac{\epsilon k}{g}\right), \quad T_{\max } \frac{\mu(g-\epsilon k)}{g(\mu+\epsilon k)}\right) .
$$

The Jacobian matrix at $(0,0)$ is now

$$
J(0,0)=\left(\begin{array}{cc}
-\mu & g+\mu \\
0 & g-\epsilon k
\end{array}\right),
$$

so the eigenvalues are $\lambda_{-}=-\mu$ and $\lambda_{*}=g-\epsilon k$, which means that the origin may be a saddle or an attractor depending on the sign of $g-\epsilon k$.

If $g \leq \epsilon k$, then the equilibrium point (42) is not in quadrant I or it is the origin. In this case, the origin is the only equilibrium point of the system, and it is an attractor. Hence, starting the solution trajectory at $\left(K_{M}, K_{M}\right)$ in the third subinterval, it will tend toward $(0,0)$, but upon reentering the second subinterval the trajectory will then tend toward $\left(T_{\max }, T_{\max }\right)$, but upon reentering the third subinterval the trajectory will then tend toward $(0,0)$, and so on, so that the solution trajectory will oscillate about the line $W=K_{M}$. Hence there will be an attracting "equilibrium point" with $W$ coordinate $K_{M}$ inside $\Omega$. Figure 5(a) illustrates this case which has traveling wave profiles that oscillate significantly behind the wave front.

If $g>\epsilon k$, then the equilibrium point (42) is in quadrant I, and the Jacobian matrix at the nonzero equilibrium point (42) is

$$
\left(\begin{array}{cc}
\frac{-\mu(g+\mu)}{\epsilon k+\mu} & \epsilon k+\mu \\
\frac{-\mu(g-\epsilon k)}{\epsilon k+\mu} & 0
\end{array}\right)
$$



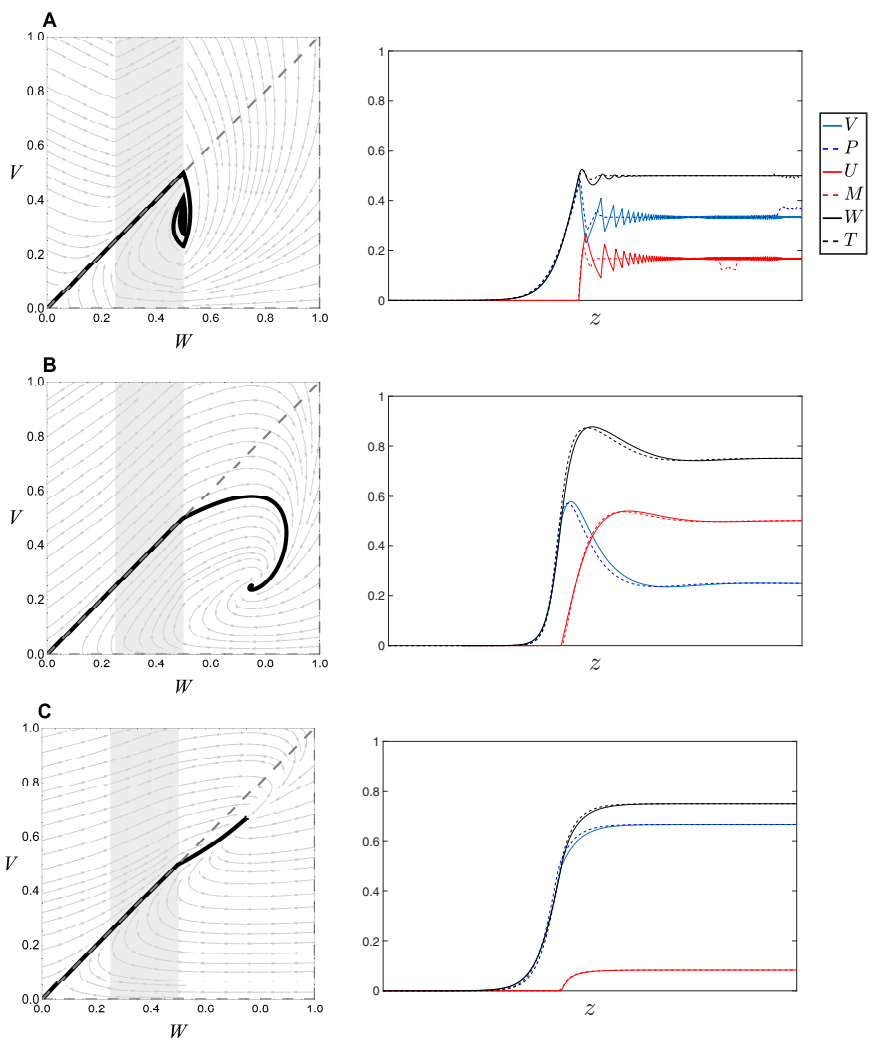

FIG. 5. (Left) Phase portrait of the system (19) with $n \rightarrow \infty$ and condition $K_{P}<K_{M}$. The colors and styles of the curves are as described in Figure 4. The gray shaded region denotes the interval $\left[K_{P}, K_{M}\right)$. (Right) The corresponding traveling wave profile of the solution trajectory in traveling wave coordinate $z$ (analytical profiles: $V, U, W$; numerical profiles: $P, M, T$ ). Parameters $T_{\max }=1, \epsilon=1, k=0.5, K_{P}=0.25, K_{M}=0.5$, and (a) $g=0.25, \mu=0.25$; (b) $g=2, \mu=0.25$; (c) $g=2, \mu=4$.

thus the determinant is $\mu(g-\epsilon k)>0$, and the trace is $-\mu(g+\mu) /(\epsilon k+\mu)<0$, so the equilibrium point (42) is an attractor.

If the $W$-coordinate of the nonzero equilibrium point (42) is in the first or second subinterval, then the solution trajectory reenters the second subinterval, and the behavior of the system is similar to the case when $g \leq \epsilon k$ (Figure 5(a)).

If the $W$-coordinate of the nonzero equilibrium point (42) is in the third subinterval, then to determine what type of attractor the equilibrium point is, we examine the sign of $(\operatorname{tr} J)^{2}-4 \operatorname{det} J$. There is a stable spiral (Figure $\left.5(\mathrm{~b})\right)$ if

$$
g-\epsilon k>\frac{\mu}{4}\left(\frac{g+\mu}{\epsilon k+\mu}\right)^{2},
$$

and the resulting traveling wave profiles have a prominent bump at the wave front. There is a stable node (Figure 5(c)) if

$$
g-\epsilon k<\frac{\mu}{4}\left(\frac{g+\mu}{\epsilon k+\mu}\right)^{2}
$$

and the resulting traveling wave profiles are monotonic and do not have a bump. 
3.5.2.2. Subcase: $\boldsymbol{K}_{\boldsymbol{P}}>\boldsymbol{K}_{\boldsymbol{M}}$. If instead we consider $K_{P}>K_{M}$, then (40b) can be rewritten as

$$
\frac{d V}{d z}=g\left(1-\frac{W}{T_{\max }}\right) V+ \begin{cases}k(W-V), & W \in\left[0, K_{M}\right), \\ -\epsilon k V+k(W-V), & W \in\left[K_{M}, K_{P}\right), \\ -\epsilon k V, & W \in\left[K_{P}, T_{\max }\right] .\end{cases}
$$

The equations in the first and third subintervals are the same as in the previous subcase (41) while the equation in the second subinterval is different. Therefore, in the first subinterval we have the same result as in the first subinterval in subsection 3.5.2.1. In particular, the trajectory leaving the saddle at $(0,0)$ will coincide with the line $\{(W, V): V=W\}$ for the entire subinterval, and the trajectory will be picked up going into the second subinterval at the point $(W, V)=\left(K_{M}, K_{M}\right)$.

Second subinterval $\left[K_{M}, K_{P}\right)$. For the equations defined in the second subinterval, the equilibrium points are $(W, V)=(0,0)$ and

$$
(W, V)=\left(T_{\max } \frac{g(k+\mu)-\epsilon k \mu}{g(k+\mu)}, \quad T_{\max } \frac{g(k+\mu)-\epsilon k \mu}{g(k+\epsilon k+\mu)}\right) .
$$

If $g(k+\mu) \leq \epsilon k \mu$, then the nonzero equilibrium point (48) is not in quadrant I or coincides with the origin in the case of equality. Thus the only equilibrium point in quadrant $\mathrm{I}$ in this case is $(0,0)$. The Jacobian matrix at $(0,0)$ is

$$
J(0,0)=\left(\begin{array}{cc}
-\mu & g+\mu \\
k & g-\epsilon k-k
\end{array}\right)
$$

which has determinant $\epsilon k \mu-g(k+\mu) \geq 0$ and trace $g-(\epsilon k+k+\mu)$. The trace is nonpositive, since all the parameters are positive, and starting with the assumption $g(k+\mu) \leq \epsilon k \mu$,

$$
g(k+\mu) \leq \epsilon k \mu \leq \epsilon k \mu+\epsilon k^{2}+k^{2}+2 k \mu+\mu^{2}=(\epsilon k+k+\mu)(k+\mu),
$$

and then dividing by $k+\mu$ (which is positive) implies that $g \leq \epsilon k+k+\mu$. Therefore the origin is an attractor. The trajectory that starts in the second subinterval at the point $(W, V)=\left(K_{M}, K_{M}\right)$ gets drawn back into the first subinterval and will oscillate about the line $W=K_{M}$, similar to the case in subsection 3.5.2.1 when $g \leq \epsilon k$. Figure 6 illustrates this case where there is an attracting "equilibrium point" with $W$-coordinate $K_{M}$ inside $\Omega$.

If $g(k+\mu)>\epsilon k \mu$, the origin is a saddle since the determinant of the Jacobian matrix $J(0,0)$ is negative. Also, the nonzero equilibrium point (48) is in $\Omega$ since the condition in (37) is satisfied with the assumption that all of the parameters are positive. The Jacobian matrix at the nonzero equilibrium point (48) is

$$
\left(\begin{array}{cc}
\frac{-(g+\mu)(k+\mu)}{k+\epsilon k+\mu} & \frac{\mu(k+\epsilon k+\mu)}{k+\mu} \\
\frac{-(g-k-\epsilon k)(k+\mu)}{k+\epsilon k+\mu} & \frac{-k(k+\epsilon k+\mu)}{k+\mu}
\end{array}\right)
$$

which has the determinant $g(k+\mu)-\epsilon k \mu>0$ and trace

$$
-\frac{k^{3}\left(1+\epsilon^{2}\right)+k^{2}(3+2 \epsilon) \mu+3 k \mu^{2}+\mu^{3}+g(k+\mu)^{2}}{(k+\mu)(k+\epsilon k+\mu)}<0,
$$



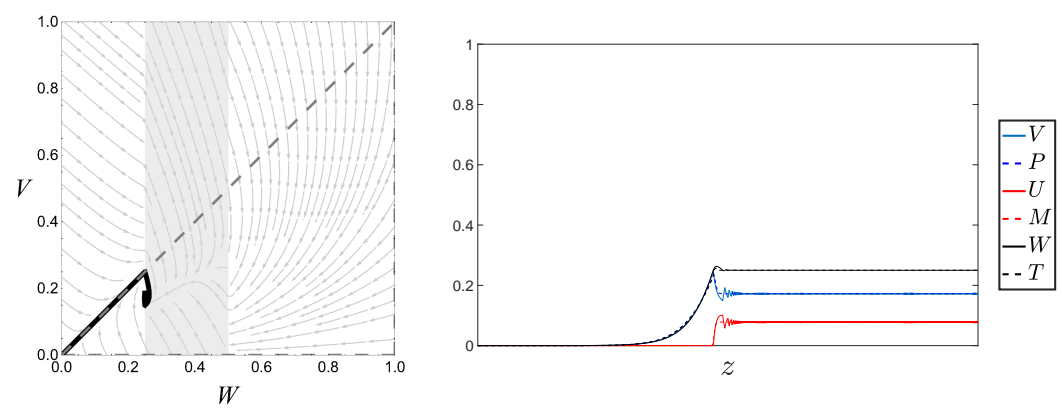

FIG. 6. (Left) Phase portrait of the system (19) with $n \rightarrow \infty$ and conditions $K_{P}>K_{M}$ and $g(k+\mu) \leq \epsilon k \mu$. The colors and styles of the curves are as described in Figure 4. The gray shaded region denotes the interval $\left[K_{M}, K_{P}\right)$. (Right) The corresponding traveling wave profile of the solution trajectory in traveling wave coordinate $z$ (analytical profiles: $V, U, W$; numerical profiles: $P, M, T)$. Parameters $T_{\max }=1, \epsilon=1, k=0.5, \mu=0.25, K_{P}=0.5, K_{M}=0.25$ and $g=0.15$.

implying that the nonzero equilibrium point (48) is an attractor. The type of attractor depends on the sign of $(\operatorname{tr} J)^{2}-4 \operatorname{det} J$, which will result in different behavior in the solution trajectory. Furthermore, the solution trajectory behavior depends on which subinterval the nonzero equilibrium point (48) is in.

If the $W$-coordinate of the nonzero equilibrium point (48) is in the first subinterval $\left[0, K_{M}\right)$, then the solution trajectory is drawn back into the first subinterval and will oscillate significantly about the line $W=K_{M}$, similar to the behavior seen in Figure 6 .

If the $W$-coordinate of the nonzero equilibrium point (48) is in the second subinterval $\left[K_{M}, K_{P}\right)$, and the attractor is a stable spiral (Figure $7(\mathrm{a})$ ), then the resulting traveling wave profiles have a prominent bump at the wave front. If the attractor is a stable node (Figure 7(b)), then the traveling wave profiles also have a prominent bump at the wave front when the solution trajectory quickly changes paths when entering the second subinterval $\left[K_{M}, K_{P}\right)$.

If the $W$-coordinate of the nonzero equilibrium point (48) is in the third subinterval $\left[K_{P}, T_{\max }\right]$, then the solution trajectory enters the third subinterval, and we must examine the dynamical system there.

Third subinterval $\left[K_{P}, T_{\max }\right]$. Since the equation for $\frac{d V}{d z}$ in the third subinterval in this subsection (47) and the previous subsection (41) is the same, all of the analysis for the third subinterval in subsection 3.5.2.1 is relevant.

To travel from the second subinterval into the third subinterval along a solution trajectory, recall that the following two inequalities must hold:

$$
g(k+\mu)>\epsilon k \mu, \quad K_{P}<T_{\max } \frac{g(k+\mu)-\epsilon k \mu}{g(k+\mu)} .
$$

For the trajectory to stay in the third subinterval, recall from subsection 3.5.2.1 that these two inequalities must hold:

$$
g>\epsilon k, \quad K_{P}<T_{\max }\left(1-\frac{\epsilon k}{g}\right) .
$$

In fact, if both inequalities in (54) hold, then both inequalities in (53) hold, assuming that all the parameters are positive.

In the situation where the solution trajectory enters the third subinterval but the $W$-coordinate of the nonzero equilibrium point (42) is not in the third subinterval, 

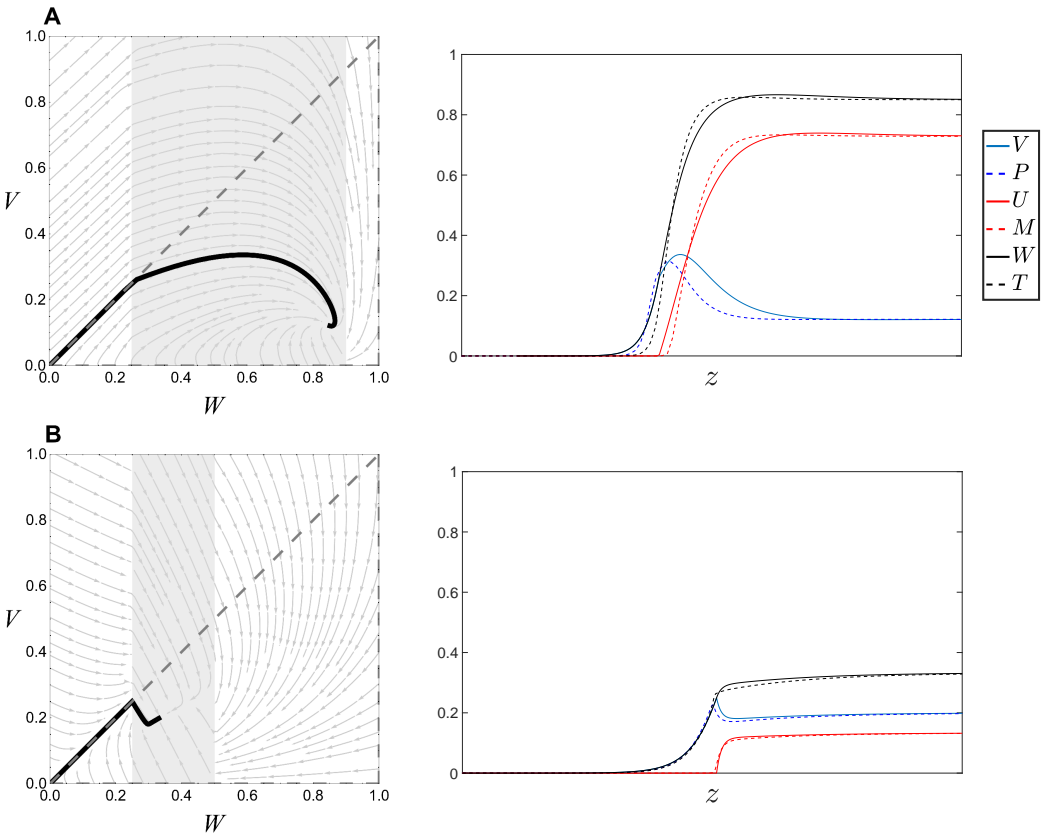

FIG. 7. (Left) Phase portrait of the system (19) with $n \rightarrow \infty$ and conditions $K_{P}>K_{M}$ and $g(k+\mu)>\epsilon k \mu$. The colors and styles of the curves are as described in Figure 4. The gray shaded region denotes the interval $\left[K_{M}, K_{P}\right)$. (Right) The corresponding traveling wave profile of the solution trajectory in traveling wave coordinate $z$ (analytical profiles: $V, U, W$; numerical profiles: $P, M, T)$. Parameters $T_{\max }=1, k=0.5, \mu=0.25, K_{M}=0.25$, and (a) $g=10, \epsilon=9, K_{P}=0.9$; (b) $g=0.25, \epsilon=1, K_{P}=0.5$.

then the trajectory will oscillate significantly around the vertical line $W=K_{P}$, similar to the behavior in Figures 5(a) and 6.

If the solution trajectory enters the third subinterval and the $W$-coordinate of the nonzero equilibrium point (42) is in the third subinterval, then the equilibrium point is a stable spiral if condition (45) holds. The phase portraits and wave profiles in this case when $K_{P}>K_{M}$ (Figure 8(a)) are very similar to when $K_{P}<K_{M}$ in the previous subsection (Figure 5(b)) for the otherwise same set of parameter values. The main difference is that the slope of the solution trajectory in the second subinterval is less steep when $K_{P}>K_{M}$, resulting in a less steep slope in the traveling wave profile fronts.

If condition (46) holds instead, then the nonzero equilibrium point in the third subinterval is a stable node. Similarly to systems with a stable spiral, the phase portraits when $K_{P}>K_{M}$ (Figure 8(b)) are very similar to when $K_{P}<K_{M}$ in the previous subsection (Figure $5(\mathrm{c})$ ) for the otherwise same set of parameter values. It is also the case that the slope of the solution trajectory in the second subinterval is less steep when $K_{P}>K_{M}$, resulting in a less steep slope in the traveling wave profile fronts.

3.5.2.3. Subcase: $\boldsymbol{K}_{\boldsymbol{P}}=\boldsymbol{K}_{\boldsymbol{M}}$. If $K_{P}=K_{M}$, then (40b) can be rewritten as

$$
\frac{d V}{d z}=g\left(1-\frac{W}{T_{\max }}\right) V+ \begin{cases}k(W-V), & W \in\left[0, K_{P}\right), \\ -\epsilon k V, & W \in\left[K_{P}, T_{\max }\right] .\end{cases}
$$

The equation in the first subinterval is the same as in the first subinterval in the previous two subcases ((41) and (47)), and the equation in the second subinterval 

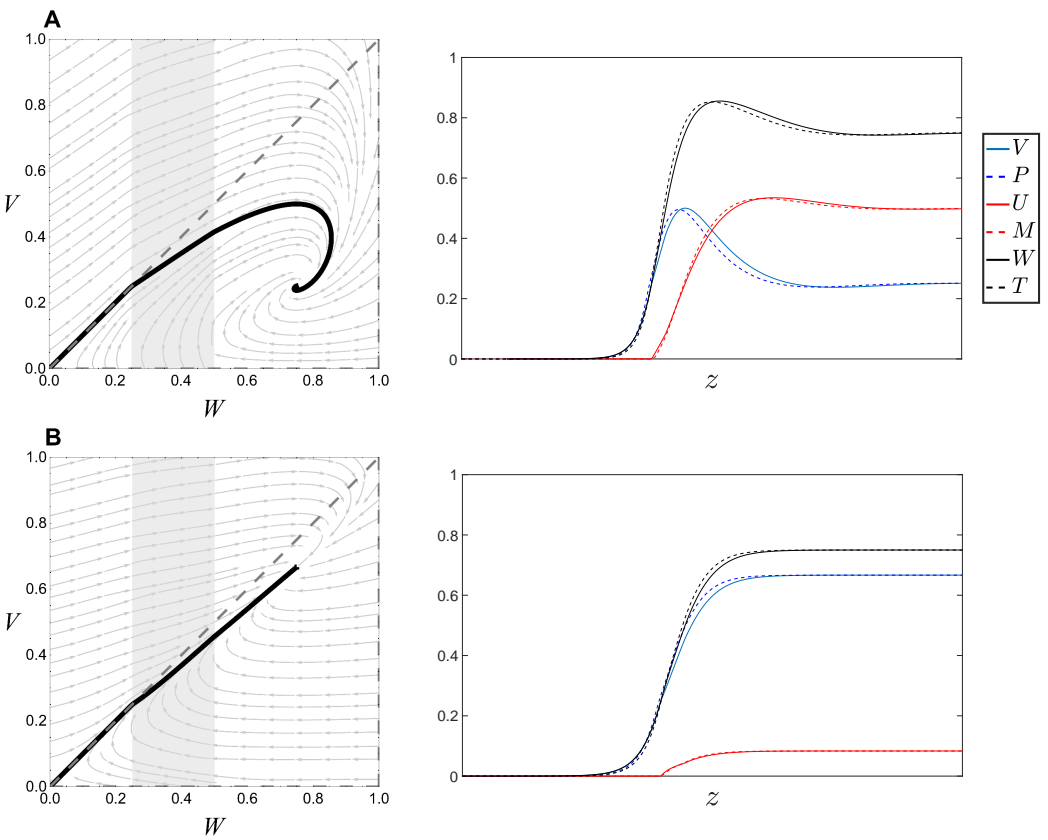

FIG. 8. (Left) Phase portrait of the system (19) with $n \rightarrow \infty$ and conditions $K_{P}>K_{M}$ and (54). The colors and styles of the curves are as described in Figure 4. The gray shaded region denotes the interval $\left[K_{M}, K_{P}\right)$. (Right) The corresponding traveling wave profile of the solution trajectory in traveling wave coordinate $z$ (analytical profiles: $V, U, W$; numerical profiles: $P, M$, T). Parameters $T_{\max }=1, \epsilon=1, k=0.5, K_{P}=0.5, K_{M}=0.25$, and (a) $g=2, \mu=0.25$ (cf. Figure 5(b)); (b) $g=2, \mu=4$ (cf. Figure 5(c)).

is the same as in the third subinterval in the previous two subcases $((41)$ and (47)). Therefore, a solution trajectory of the system with $K_{P}=K_{M}$ follows the behavior of the previous two subcases, where the middle subinterval is not included. Representative phase portraits and traveling wave profiles would look most similar to Figure 5 .

3.5.3. Case: $1 \leq \boldsymbol{n}<\infty$. If we set $1 \leq n<\infty$, then we have the general system (19). The origin $(W, V)=(0,0)$ is one equilibrium point, and the other equilibrium points are such that

$$
V=\frac{\mu W}{g\left(1-\frac{W}{T_{\max }}\right)+\mu}
$$

and $W$ satisfies a polynomial equation

$$
A W^{2 n+1}+B W^{2 n}+C W^{n+1}+D W^{n}+E W+F=0,
$$

where

$$
\begin{aligned}
& A=g \mu, \\
& B=\mu T_{\max }(\epsilon k-g) \\
& C=g\left((k+\mu) K_{P}^{n}+\mu K_{M}^{n}\right), \\
& D=T_{\max }\left(\epsilon k \mu K_{P}^{n}-g\left((k+\mu) K_{P}^{n}+\mu K_{M}^{n}\right)\right),
\end{aligned}
$$

Copyright (C) by SIAM. Unauthorized reproduction of this article is prohibited. 


$$
\begin{aligned}
& E=g K_{P}^{n} K_{M}^{n}(k+\mu), \\
& F=-T_{\max } g K_{P}^{n} K_{M}^{n}(k+\mu) .
\end{aligned}
$$

Since all of the parameters are positive, then coefficients $A, C, E>0$ and $F<0$. From Descartes' rule of signs, there is at least one positive root. To examine the possible roots of (57), we perturb the system from $\mu=0$.

When $\mu=0$, the nonzero equilibrium point is

$$
(W, V)=\left(T_{\max }, \quad \frac{K_{P}^{n} T_{\max }\left(K_{M}^{n}+T_{\max }^{n}\right)}{K_{M}^{n} K_{P}^{n}+T_{\max }^{n}\left((1+\epsilon) K_{P}^{n}+\epsilon T_{\max }^{n}\right)}\right) .
$$

The Jacobian matrix at this nonzero equilibrium point is lower triangular and has two negative eigenvalues

$$
-\left(\frac{k K_{P}^{n}}{K_{P}^{n}+T_{\max }^{n}}+\frac{k T_{\max }^{n} \epsilon}{K_{M}^{n}+T_{\max }^{n}}\right), \quad-\frac{g K_{P}^{n}\left(K_{M}^{n}+T_{\max }^{n}\right)}{K_{M}^{n} K_{P}^{n}+T_{\max }^{n}\left((1+\epsilon) K_{P}^{n}+\epsilon T_{\max }^{n}\right)}
$$

and is thus a stable node. One of the linearized eigenvectors is $[0,1]^{\top}$, and the slope of the other eigenvector determines whether the wave profile will have a prominent bump (slope $<0)$ (Figure 9(a)) or will be monotonic (slope $\geq 0$ ).

We observe that the solutions of the polynomial equation (57) are $W=T_{\max }$, which corresponds with the $W$-coordinate of the equilibrium point (59), and $W=$ $\left(-K_{M}^{n}\right)^{1 / n}$, which is either negative or imaginary depending on whether $n$ is odd or even. Since we require $W \geq 0$ for physically relevant solutions, we only investigate perturbations from the nonnegative real root $W=T_{\max }$. In other words, we investigate how the equilibrium point (59) moves as $\mu$ increases.

Substituting the regular perturbation expansion

$$
W(\mu)=\sum_{r=0}^{\infty} W_{r} \mu^{r}
$$

into polynomial (57) results in a power series in $\mu$. Requiring each term to vanish, the first three coefficients of the series (61) are

$$
\begin{aligned}
W_{0}= & T_{\max }, \\
W_{1}= & -\frac{\epsilon T_{\max }^{n+1}\left(K_{P}+T_{\max }^{n}\right)}{g K_{P}\left(K_{M}+T_{\max }^{n}\right)}, \\
W_{2}= & \epsilon T_{\max }^{n+1}\left(K_{P}+T_{\max }^{n}\right)\left[g\left(K_{M}+T_{\max }^{n}\right)^{2}\left(K_{P}+T_{\max }^{n}\right)\right. \\
& \left.+n \epsilon k T_{\max }^{n}\left(T_{\max }^{2 n}+K_{M}\left(K_{P}+2 T_{\max }^{n}\right)\right)\right] / g^{2} k K_{P}^{2}\left(K_{M}+T_{\max }^{n}\right)^{3} .
\end{aligned}
$$

Assuming the perturbation $\mu$ is small, the coefficient that determines whether $W(\mu)$ increases or decreases is $W_{1}$. Thus since $W_{1}$ is negative, $W(\mu)$ decreases as $\mu$ increases.

Considering (56) as a function of $\mu$, the partial derivative

$$
\frac{\partial V}{\partial \mu}=\frac{g\left(1-\frac{W}{T_{\max }}\right) W}{\left(g\left(1-\frac{W}{T_{\max }}\right)+\mu\right)^{2}}>0
$$

implying that the $V$-coordinate of the nonnegative equilibrium point (56) increases as $\mu$ increases. Furthermore, as $\mu \rightarrow \infty$, the $V$-coordinate of the nonnegative equilibrium 

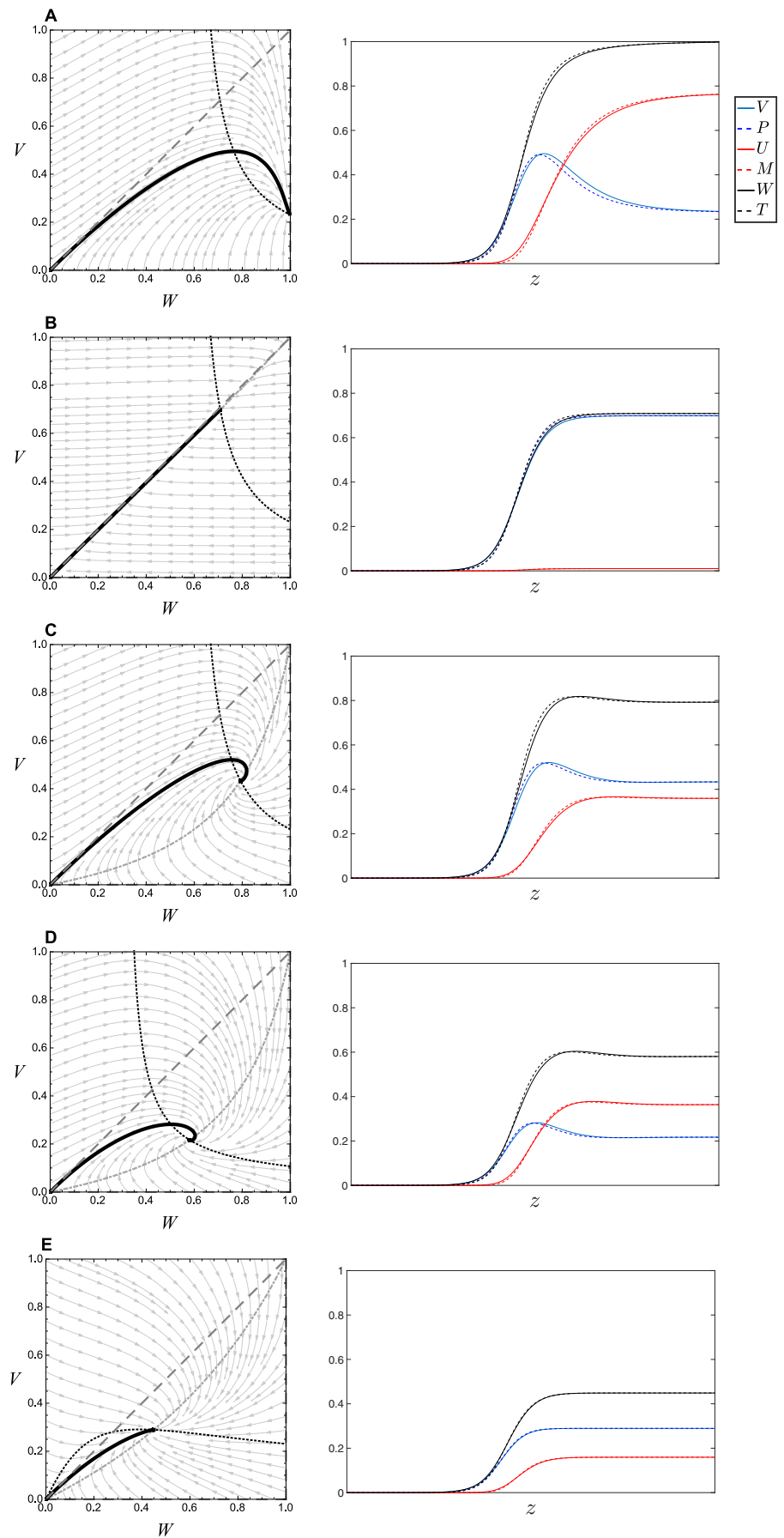

FIG. 9. (Left) Phase portrait of the system (19) with $n=1$. The colors and styles of the curves are as described in Figure 4. (Right) The corresponding traveling wave profile of the solution trajectory in traveling wave coordinate $z$ (analytical profiles: $V, U, W$; numerical profiles: $P, M$, $T$ ). Parameters $T_{\max }=1, k=0.5, K_{P}=0.25, K_{M}=0.5$, and (a) $g=1, \mu=0, \epsilon=1$; (b) $g=1$, $\mu=20, \epsilon=1$; (c) $g=1, \mu=0.25, \epsilon=1$; (d) $g=1, \mu=0.25, \epsilon=2.5$; (e) $g=0.25, \mu=0.25, \epsilon=1$.

Copyright (c) by SIAM. Unauthorized reproduction of this article is prohibited. 
point (56) tends to $W$ in the limit (Figure 9(b)). Thus the nonnegative equilibrium point defined by (56)-(57) is in the positively invariant region $\Omega$ for $\mu>0$. Furthermore from the perturbation analysis, there are no other interior equilibrium points that arise.

Recall from subsection 3.1 that the origin is a saddle, and by reasoning at the beginning of subsection 3.5, the nonnegative equilibrium point defined by (56)-(57) is an attractor. Variations in the shape of the traveling wave profile thus depend on the type of attractor that the equilibrium point is. In general, if the nonnegative equilibrium point is a stable spiral, the wave profile will have a prominent bump (Figure 9(c),(d)) and if it is a stable node, the wave profile will be monotonic (Figure $9(\mathrm{e})$ ). The density of the proliferating and migrating cells in the center of the tumor core (as $z \rightarrow \infty$ ) is given by the nonnegative equilibrium point, and thus we can find cases where there are more proliferating cells in the center (Figure $9(\mathrm{c}),(\mathrm{e})$ ) or more migrating cells in the center (Figure 9(d)).

4. Discussion. We analyzed the speed and shape of traveling wave solutions of a mathematical model of GBM growth in which tumor cell motility and cell proliferation are considered as separate processes. The model is based on the "Go or Grow" hypothesis in which an individual cell is either primarily migrating or primarily proliferating, and a phenotypic switch is responsible for the transformation of a cell from one population to the other. We examined an extreme form of this hypothesis where the migrating cells do not proliferate at all and the proliferating cells do not migrate at all, resulting in a two-equation system of partial differential equations.

In general, establishing the existence of traveling wave solutions in a nonmonotone and nonlinear population reaction-diffusion based model is nontrivial. Based on extensive simulation results, Lewis and van den Driessche [16] conjectured that their competition model involving fertile and a sterile insect populations may admit traveling waves with speed highly dependent on the sterile population density. Specifically, they found that if the sterile population density is sufficiently low, the traveling wave advances, and when the sterile density exceeds a threshold, the wave reverses direction.

In a multipopulation model, the "linear determinacy" conjecture equates full nonlinear model spread rates with the spread rates computed from linearized systems with the linearization carried out around the leading edge of the invasion (Castillo-Chavez et al. [3]). Lewis et al. [15] derived a set of sufficient conditions for linear determinacy in spatially explicit two-species competition models. These conditions can be interpreted as requiring sufficiently large dispersal of the invader relative to dispersal of the out-competed resident and sufficiently weak interactions between the resident and the invader. When these conditions are not satisfied, spread rate may exceed linearly determined predictions.

We determined a minimum wave speed (15) for traveling wave solutions, and the numerically observed wave speed was in general greater than or equal to the minimum wave speed. The parameter $k$ appeared to have the largest effect on the difference in the analytic versus numerical wave speeds. Oftentimes in simpler equations that give rise to traveling waves (such as the Fisher-Kolmogorov equation), the minimum wave speed and the numerically observed wave speed coincide, so it may be due to the nonlinearities of the transition functions that the speeds do not coincide for the model presented here. It could also be due to the two-equation nature of the model, as it has been previously shown that a cooperative system may have the numerically observed 
wave speed exceeding the analytic minimum speed due to the changing interacting species (Li et al. [17, Example 4.1]). Similar situations can also be found for integrodifferential equation cooperative systems (Hu et al. [13]). Since the minimum wave speed (15) was found after linearization of the system (7) and does not contain all of the parameters that are included in the model (4), further study should be done to investigate whether a more accurate expression can be found for the minimum wave speed. Furthermore, an interesting future direction is to determine what features of the kinetics in the model system are required in order to have a minimum wave speed or no minimum wave speed, noting that our system is neither cooperative or competitive. There are also opportunities of extending our approach to the study of species invasion, resistance strain development in disease, and cancer progression with treatment, for example.

To investigate the shape of the traveling wave fronts, we adapted the method of Canosa [2] to obtain an approximation of the traveling wave solution. Canosa's method involves finding an asymptotic expansion for large wave speed, which we expect to hold under certain stages of GBM. We investigated the conditions under which simulations of equations (16) and (18) match well. Figure 10 displays the simulations for $U(z)$ and $V(z)$ for various wave speeds. For small values of the wave speed, $c$, (left; $c=0.01$ ), it is apparent that the simulations do not agree. For larger values of wave speed (right; $c=0.05$ ) we can see that the approximation is valid. While studying the resulting approximate system (19), we found a positively invariant region in which no periodic orbits exist and in which the unstable manifold of the saddle $(0,0)$ has nonempty intersection. One conjecture that has yet to be proven pertains to the monotonicity of the $V$-nullcline (26).

Depending on the value of parameters $n, K_{P}$, and $K_{M}$, we explored different results regarding the number and location of interior equilibrium points within the positively invariant region. Conditions were found that determined whether an interior equilibrium point was a stable spiral, which results in traveling wave profiles that
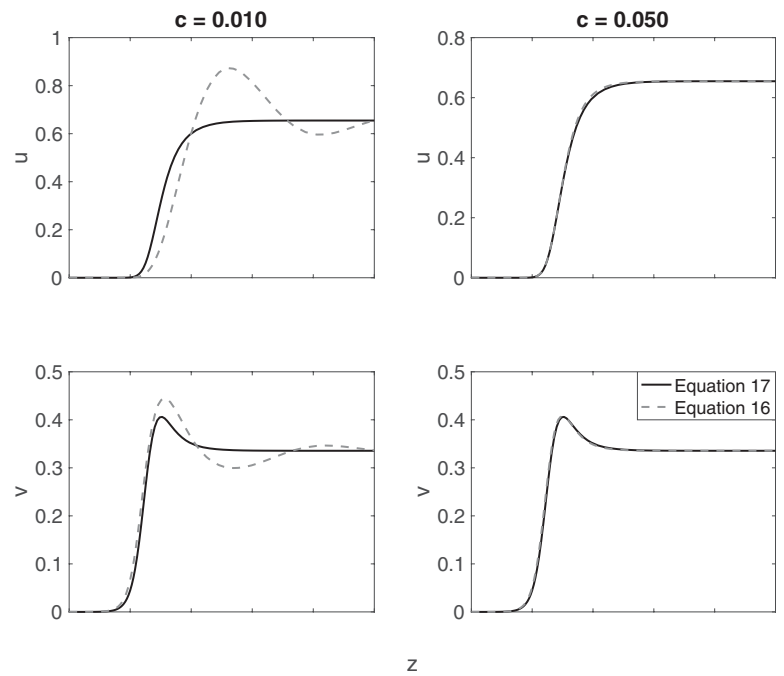

FIG. 10. Comparison of equations (16) and (18) for various wave speeds, c. On the left, a small wave speed for which the approximation is not accurate. On the right, a large wave speed for which there is good agreement between the equations. The base parameters are the same as those from Figure 1: $D=5 \times 10^{-4}, g=1, k=1, \mu=0.005, K_{m}=K_{p}=0.5$, and $\epsilon=1$. 
have a prominent bump at the wave front, or a stable node, which results in traveling wave profiles that are monotonic. We visually compared the traveling wave profiles that were obtained from the approximate traveling wave solution with numerical simulations (Figures 4 to 9, right-side panel), and found that overall there was very good agreement.

Biologically, the results of this study imply that the parameter values play a large role in determining which cell population has a larger density within the tumor core and whether there is a clumping of certain cells near the moving boundary. When there is a stable spiral in the dynamical system, this corresponds to a higher density of cells near the moving boundary as opposed to a little further behind the wave front. When there is a stable node in the dynamical system, the density of cells near the moving boundary is the same as the density of cells in the tumor core.

While all the possibilities for different wave front shapes in the cases when $n=0$ and $n \rightarrow \infty$ have been fully explored, the case when $1 \leq n<\infty$ presents problems in the ease of writing down explicit analytical expressions for categorizing wave front shapes. However, setting $\mu=0$ and then perturbing from the resulting system gives the conclusion that there is only one equilibrium point in the positively invariant region that corresponds with the density of cells in the center of the tumor core. Classification of this equilibrium point determines the shape of the traveling wave front, as described two paragraphs back. Further study includes determining whether the different profile shapes are seen biologically and if they are stable.

\section{REFERENCES}

[1] A. Q. Cai, K. A. Landman, And B. D. Hughes, Multi-scale modeling of a wound-healing cell migration assay, J. Theoret. Biol., 245 (2007), pp. 576-594, https://doi.org/10.1016/j.jtbi. 2006.10.024.

[2] J. CANosa, On a nonlinear diffusion equation describing population growth, IBM J. Res. Dev., 17 (1973), pp. 307-313, https://doi.org/10.1147/rd.174.0307.

[3] C. Castillo-Chavez, B. Li, And H. Wang, Some recent developments on linear determinancy, Math. Biosci. Eng., 10 (2013), pp. 1419-1436, https://doi.org/10.3934/mbe.2013.10.1419.

[4] A. Chauviere, L. Preziosi, and H. Byrne, A model of cell migration within the extracellular matrix based on a phenotypic switching mechanism, Math. Med. Biol., 27 (2010), pp. 255-281, https://doi.org/10.1093/imammb/dqp021.

[5] P. D. Dale, J. A. Sherratt, and P. K. Maini, Role of fibroblast migration in collagen fiber formation during fetal and adult dermal wound healing, Bull. Math. Biol., 59 (1997), pp. 1077-1100, https://doi.org/10.1007/BF02460102.

[6] A. Farin, S. O. Suzuki, M. Weiker, J. E. Goldman, J. N. Bruce, and P. Canoll, Transplanted glioma cells migrate and proliferate on host brain vasculature: A dynamic analysis, Glia, 53 (2006), pp. 799-808, https://doi.org/10.1002/glia.20334.

[7] P. Gerlee And S. Nelander, Travelling wave analysis of a mathematical model of glioblastoma growth, Math. Biosci., 276 (2016), pp. 75-81, https://doi.org/10.1016/j.mbs.2016.03.004.

[8] A. Giese, R. Buerkvig, M. Berens, and M. Westphal, Cost of migration: Invasion of malignant gliomas and implications for treatment, J. Clin. Oncol., 21 (2003), pp. 1624-1636, https://doi.org/10.1200/JCO.2003.05.063.

[9] A. Giese, L. Kluwe, B. Laube, And M. E. Berens, Migration of human glioma cells on myelin, Neurosurgery, 38 (1996), pp. 755-764, https://doi.org/10.1227/00006123199604000-00026.

[10] A. Giese, M. A. Loo, D. Tran, S. W. Haskett, and B. M. E. Coons, Dichotomy of astrocytoma migration and proliferation, Int. J. Cancer, 67 (1996), pp. 275-282, https: //doi.org/10.1002/(SICI)1097-0215(19960717)67:2〈275::AID-IJC20〉3.0.CO;2-9.

[11] J. Godlewski, M. O. Nowicki, A. Bronisz, G. Nuovo, J. Palatini, M. De Lay, J. Van Brocklyn, M. C. Ostrowski, E. A. Chiocca, and S. E. Lawler, MicroRNA-451 regulates LKB1/AMPK signaling and allows adaptation to metabolic stress in glioma cells, Mol. Cell, 37 (2010), pp. 620-632, https://doi.org/10.1016/j.molcel.2010.02.018.

Copyright $@$ by SIAM. Unauthorized reproduction of this article is prohibited. 
[12] H. Hatzikirou, D. Basanta, M. Simon, K. Schaller, and A. Deutsch, "Go or Grow": The key to the emergence of invasion in tumour progression?, Math. Med. Biol., 29 (2012), pp. 49-65, https://doi.org/10.1093/imammb/dqq011.

[13] C. Hu, Y. Kuang, B. Li, And H. Liu, Spreading speeds and traveling wave solutions in cooperative integral-differential systems, Discrete Contin. Dyn. Syst. Ser. B, 20 (2015), pp. 1663-1684, https://doi.org/10.3934/dcdsb.2015.20.1663.

[14] K. A. Landman, A. Q. CaI, And B. D. Hughes, Travelling waves of attached and detached cells in a wound-healing cell migration assay, Bull. Math. Biol., 69 (2007), pp. 2119-2138, https://doi.org/10.1007/s11538-007-9206-0.

[15] M. A. Lewis, B. LI, AND H. F. WeinBerger, Spreading speed and linear determinacy for two-species competition models, J. Math. Biol., 45 (2002), pp. 219-233, https://doi.org/ $10.1007 / \mathrm{s} 002850200144$.

[16] M. A. Lewis AND P. VAN DEN Driessche, Waves of extinction from sterile insect release, Math. Biosci., 116 (1993), pp. 221-247, https://doi.org/10.1016/0025-5564(93)90067-K.

[17] B. Li, H. F. Weinberger, And M. A. Lewis, Spreading speeds as slowest wave speeds for cooperative systems, Math. Biosci., 196 (2005), pp. 82-98, https://doi.org/10.1016/j.mbs. 2005.03.008.

[18] A. Martínez-González, G. F. Calvo, L. A. Pérez Romasanta, and V. M. Pérez-García, Hypoxic cell waves around necrotic cores in glioblastoma: A biomathematical model and its therapeutic implications, Bull. Math. Biol., 74 (2012), pp. 2875-2896, https://doi.org/ 10.1007/s11538-012-9786-1.

[19] N. L. Martirosyan, E. M. Rutter, W. L. Ramey, E. J. Kostelich, Y. Kuang, and M. C. Preul, Mathematically modeling the biological properties of gliomas: A review, Math. Biosci. Eng., 12 (2015), pp. 879-905, https://doi.org/10.3934/mbe.2015.12.879.

[20] D. F. Newgreen, G. J. Pettet, and K. A. Landman, Chemotactic cellular migration: Smooth and discontinuous travelling wave solutions, SIAM J. Appl. Math., 63 (2003), pp. 1666-1681, https://doi.org/10.1137/S0036139902404694.

[21] A. D. Norden And P. Y. Wen, Glioma therapy in adults, Neurologist, 12 (2006), pp. 279-292, https://doi.org/10.1097/01.nrl.0000250928.26044.47.

[22] K. Pham, A. Chauviere, H. Hatzikirou, X. Li, H. M. Byrne, V. Cristini, and J. LowenGRUB, Density-dependent quiescence in glioma invasion: Instability in a simple reactiondiffusion model for the migration/proliferation dichotomy, J. Biol. Dyn., 6, Suppl. 1 (2012), pp. 54-71, https://doi.org/10.1080/17513758.2011.590610.

[23] T. Quinn And Z. Sinkala, Dynamics of prostate cancer stem cells with diffusion and organism response, BioSystems, 96 (2009), pp. 69-79, https://doi.org/10.1016/j.biosystems.2008.11. 010.

[24] E. M. Rutter, T. L. Stepien, B. J. Anderies, J. D. Plasencia, E. C. Woolf, A. C. Scheck, G. H. Turner, Q. Liu, D. Frakes, V. Kodibagkar, Y. Kuang, M. C. Preul, AND E. J. Kostelich, Mathematical analysis of glioma growth in a murine model, Sci. Rep., 7 (2017), p. 2508, https://doi.org/10.1038/s41598-017-02462-0.

[25] O. Saut, J. B. Lagaert, T. Colin, and H. M. Fathallah-Shaykh, A multilayer Grow-orGo model for GBM: Effects of invasive cells and anti-angiogenesis on growth, Bull. Math. Biol., 76 (2014), pp. 2306-2333, https://doi.org/10.1007/s11538-014-0007-y.

[26] E. Scribner and H. M. Fathallah-Shaykh, Single cell mathematical model successfully replicates key features of GBM: Go-Or-Grow is not necessary, PLoS One, 12 (2017), pp. 1-13, https://doi.org/10.1371/journal.pone.0169434.

[27] J. A. SHERRATT, Wavefront propagation in a competition equation with a new motility term modelling contact inhibition between cell populations, Proc. Roy. Soc. A, 456 (2000), pp. 2365-2386, https://doi.org/10.1098/rspa.2000.0616.

[28] J. A. Sherratt And M. A. J. Chaplain, A new mathematical model for avascular tumour growth, J. Math. Biol., 43 (2001), pp. 291-312, https://doi.org/10.1007/s002850100088.

[29] M. J. Simpson, K. A. Landman, B. D. Hughes, and D. F Newgreen, Looking inside an invasion wave of cells using continuum models: Proliferation is the key, J. Theor. Biol., 243 (2006), pp. 343-60, https://doi.org/10.1016/j.jtbi.2006.06.021.

[30] A. M. Stein, T. Demuth, D. Mobley, M. Berens, and L. M. Sander, A mathematical model of glioblastoma tumor spheroid invasion in a three-dimensional in vitro experiment, Biophy. J., 92 (2007), pp. 356-365, https://doi.org/10.1529/biophysj.106.093468.

[31] T. L. Stepien, E. M. Rutter, and Y. Kuang, A data-motivated density-dependent diffusion model of in vitro glioblastoma growth, Math. Biosci. Eng., 12 (2015), pp. 1157-1172, https: //doi.org/10.3934/mbe.2015.12.1157.

[32] C. J. Thalhauser, T. Sankar, M. C. Preul, and Y. Kunng, Explicit separation of growth and motility in a new tumor cord model, Bull. Math. Biol., 71 (2009), pp. 585-601, https: //doi.org/10.1007/s11538-008-9372-8. 
[33] A. J. Trewenack and K. A. Landman, A traveling wave model for invasion by precursor and differentiated cells, Bull. Math. Biol., 71 (2009), pp. 291-317, https://doi.org/10.1007/ s11538-008-9362-x.

[34] C. H. Wang, J. K. Rockhill, M. Mrugala, D. L. Peacock, A. Lai, K. Jusenius, J. M. Wardlaw, T. Cloughesy, A. M. Spence, R. Rockne, E. C. Alvord, and K. R. SwanSON, Prognostic significance of growth kinetics in newly diagnosed glioblastomas revealed by combining serial imaging with a novel biomathematical model, Cancer Res., 69 (2009), pp. 9133-9140, https://doi.org/10.1158/0008-5472.CAN-08-3863.

[35] H. Zhu AND C. OU, Existence of traveling wavefronts for Sherratt's avascular tumor model, Chaos Solitons Fractals, 44 (2011), pp. 218-225, https://doi.org/10.1016/j.chaos.2011.01. 011.

[36] H. ZHU, W. YUAN, AND C. OU, Justification for wavefront propagation in a tumour growth model with contact inhibition, Proc. Roy. Soc. A, 464 (2008), pp. 1257-1273, https://doi. org/10.1098/rspa.2007.0097.

Copyright $@$ by SIAM. Unauthorized reproduction of this article is prohibited. 